\title{
Orbitofrontal and Anterior Cingulate Cortex Neurons Selectively Process Cocaine-Associated Environmental Cues in the Rhesus Monkey
}

\author{
Eun Ha Baeg, Mark E. Jackson, Hank P. Jedema, and Charles W. Bradberry \\ Departments of Psychiatry and Neuroscience, University of Pittsburgh, Veterans Affairs Pittsburgh Health Services, Pittsburgh, Pennsylvania 15261
}

\begin{abstract}
Encounters with stimuli associated with drug use are believed to contribute to relapse. To probe the neurobiology of environmentally triggered drug use, we have conducted single-unit recordings in rhesus monkeys during presentation of two distinct types of drug paired cues that differentially support drug-seeking. The animals were highly conditioned to these cues via exposure during self-administration procedures conducted over a 4 year period. The cues studied were a discriminative cue that signaled response-contingent availability of cocaine, and a discrete cue that was temporally paired with the cocaine infusion $(0.1$ or $0.5 \mathrm{mg} / \mathrm{kg})$. Two cortical regions consistently activated by cocaine-associated cues in human imaging studies are the orbitofrontal (OFC) and anterior cingulate cortex (ACC), though little is known about cortical neuronal activity responses to drug cues. We simultaneously recorded single-unit activity in OFC and ACC as well as in dorsal striatum in rhesus monkeys during cocaine self-administration. Dorsal striatal neurons were less engaged by drug cues than cortical regions. Between OFC and ACC, distinct functionality was apparent in neuronal responses. OFC neurons preferentially responded to the discriminative cue, consistent with a role in cue-induced drug-seeking. In contrast, the ACC did not respond more to the discriminative cue than to the discrete cue. Also distinct from the OFC, ACC showed sustained firing throughout the $18 \mathrm{~s}$ duration of the discrete cue. This pattern of sustained activation in ACC is consistent with a role in reward expectation and/or in mediating behavioral effects of discrete cues paired with drug infusions.
\end{abstract}

\section{Introduction}

Discriminative cues that indicate availability of a drug for selfadministration, and discrete cues that have been paired with the actual drug infusion, exert distinct influences over behavior. Noncontingent presentation of discriminative cues causes drugseeking more than discrete cues (Weiss et al., 2000; Di Ciano and Everitt, 2003; Yun and Fields, 2003). Thus, to gain insight into how chance encounters with cocaine-associated cues could trigger relapse, it is crucial to examine neuronal activity responses to discriminative cues. Discrete cues exert a different control over self-administration behavior in animals. Specifically, they have the ability to support very high rates of responding during selfadministration under second-order schedules of reinforcement in which they are intermittently presented, contingent on operant responses (Goldberg et al., 1981; Everitt and Robbins, 2000; Di Ciano and Everitt, 2003). Aspects of the environment associated with actual use could be serving as strong discrete cues, and thus they could play a role in maintaining drug consumption after relapse has occurred. Preferential engagement of different

\footnotetext{
Received July 6, 2009; revised Aug. 10, 2009; accepted Aug. 11, 2009.

This work was supported by grant support from the National Institute of Drug Abuse (DA10331) and the Office of Research and Development, Medical Research Service, Department of Veterans Affairs. Thanks are extended to Carl Olson, Andrew Schwartz, and Nelson Totah for comments on earlier versions of this manuscript.

Correspondence should be addressed to Charles W. Bradberry, Departments of Psychiatry and Neuroscience, University of Pittsburgh, Veterans Affairs Pittsburgh Health Services, 3501 Fifth Avenue, Room 4078, Pittsburgh, PA 15261. E-mail: bradberrycw@upmc.edu.

DOI:10.1523/JNEUROSCI.3206-09.2009

Copyright $\odot 2009$ Society for Neuroscience 0270-6474/09/2911619-09\$15.00/0
}

brain regions by these two distinct cue types could provide insight into neurobiological underpinnings of different aspects of addiction behavior, such as relapse and binge consumption.

Addiction is increasingly considered to involve maladaptations in orbitofrontal cortex (OFC) and anterior cingulate cortex (ACC) (Kalivas and Volkow, 2005; Garavan and Hester, 2007; Schoenbaum and Shaham, 2008). These regions are known to mediate cognitive function related to goal-directed behavior (Rushworth et al., 2007) and are particularly reactive to drug cues in humans (Garavan et al., 2000; Goldstein et al., 2009) and animals (Neisewander et al., 2000; McLaughlin and See, 2003). There is also a great deal of evidence that cognitive function associated with these regions is impaired in addicts (Aron and Paulus, 2007; Garavan and Hester, 2007; Robbins et al., 2008). While both OFC and ACC have been shown to be responsive to drug-associated cues, a comparison of how these areas respond to cues that regulate drug taking behavior has not been conducted at the neuronal activity level to evaluate whether there are differences in how distinct cue types are encoded.

Because the emphasis on cortical dysfunction in addiction derives primarily from clinical imaging studies, it is important to examine cortical involvement in a clinically relevant behavioral model. The animal model we have used to study neuronal activity responses to cocaine cues is the rhesus monkey with a long (4.5 years) history of cocaine self-administration associated with the specific cues examined. Cortical structure and the functions associated with cortical subregions in rhesus monkeys are highly similar to humans, and there is substantial knowledge regarding 
cortical and striatal neural correlates of cognition and reward processing. Thus, studies of neural responses to drugassociated cues in this model are particularly valuable in translational research (Passingham, 2009). To address the question of how distinct types of environmental cues differentially impact neuronal activity in OFC, ACC, and dorsal striatum, we conducted multielectrode recordings of single-unit activity in these structures simultaneously, during cue exposure and cocaine self-administration.

\section{Materials and Methods}

Animals. Two female rhesus macaques weighting $6.0-7.5 \mathrm{~kg}$ were used for these studies. Following acquisition of lever pressing behavior for food pellets, a subcutaneous vascular access port was placed mid-scapula from which a catheter extended to either the internal jugular or femoral vein. Cocaine was then introduced as the reinforcer for responding. These procedures have been previously described (Bradberry et al., 2000). Both animals had a history of reinforcement by both cocaine and a psychoactive metabolite of cocaine, cocaethylene, from a study comparing reinforcement efficacy of the two compounds using progressive ratio procedures. Cocaethylene is very similar to cocaine in that it is equipotent at inhibiting the dopamine transporter (Iyer et al., 1995) and also shares cocaine's local anesthetic properties (Tokuno et al., 2004). Cumulative exposure was as follows: animal A: cocaine, $253 \mathrm{mg} / \mathrm{kg}$, cocaethylene, $104 \mathrm{mg} / \mathrm{kg}$; animal B: $470 \mathrm{mg} / \mathrm{kg}$ cocaine, $120 \mathrm{mg} / \mathrm{kg}$ cocaethylene.

Cocaine self-administration. Animals were restrained in a chair (Primate Products, Redwood City, CA) by a collar, and placed in a behavioral chamber (Med-Associates, GA, VT)

fitted with an operant panel constructed from $1 / 4$ inch aluminum to which the chair was attached. On the panel was an operant lever, and a red and green lamp that served as visual cues. The vertical and horizontal distance between the lamps was $10.3 \mathrm{~cm}$. Animals could receive five infusions of cocaine under a fixed ratio 10 (FR10) lever press contingency, with a 10 min time out between infusions. Onset of a red lamp on the operant panel served as the discriminative cue that signaled availability of cocaine. Upon reaching the FR10 contingency, the red lamp was extinguished, and a green lamp was turned on. The green lamp discrete cue was maintained throughout the $18 \mathrm{~s}$ infusion used to flush the $0.1 \mathrm{ml}$ (infusions 1-4) or $0.5 \mathrm{ml}$ (infusion 5) bolus of cocaine through the intravenous line. There was no time limit for responding to the discriminative stimulus cue. Figure $1 a$ shows the sequence of events for the self-administration task.

Extinction procedure. After recording sessions with drug-associated cues were complete, in one animal, extinction sessions in which cocaine was replaced by saline were conducted. The green bulb used as a discrete cue was illuminated during the infusion of saline for $18 \mathrm{~s}$ if the FR10 contingency was met within $30 \mathrm{~s}$ of discriminative cue onset. The intertrial interval was shortened (random, centered on $2 \mathrm{~min}$ ) compared with the $10 \mathrm{~min}$ intertrial interval during cocaine self-administration because of the lack of drug infusion. Extinction sessions were $40 \mathrm{~min}$ in duration, and could range from 14 to 17 trials per session.

Single-unit recording. After a long history of drug self-administration, a craniotomy for placement of a recording chamber and head immobilizing apparatus was conducted under isoflurane anesthesia (1-3\%). In indicated region.

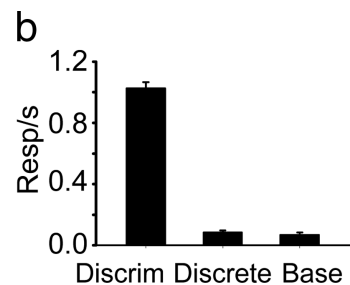

C

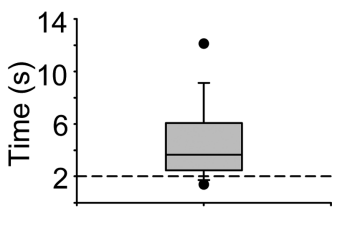

Figure 1. Behavioral paradigm and lever pressing behavior. $\boldsymbol{a}$, Self-administration and associated cues. A red lamp on the (Discrim) that signaled availability of cocaine under an FR10 contingency. Upon 列

OFC

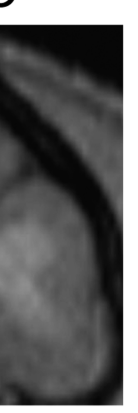

ACC

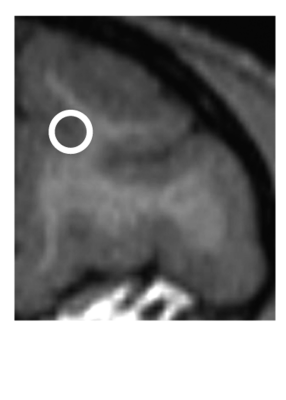

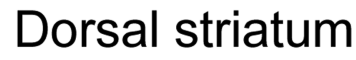

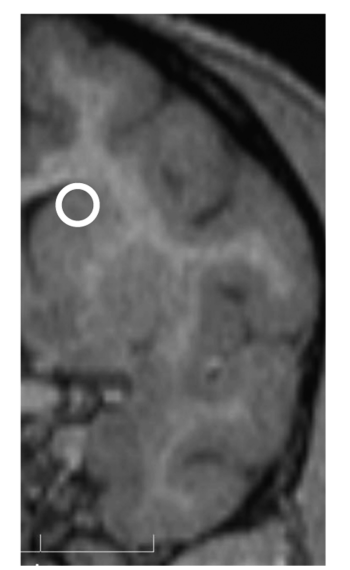

Figure 2. Recording sites. Each circle marks the approximate center on a representative MR image from animal B of the calculated recording sites. Over $90 \%$ of units in OFC and ACC were targeted to within $1 \mathrm{~mm}$ of this center. For dorsal striatum, neurons were located in a more extended area on the anterior-posterior axis, with $>80 \%$ of units targeted to within $2 \mathrm{~mm}$ of the

monkey A, a regular cannula design (Bradberry et al., 2000) was used, for animal B, a commercial recording cylinder was used (Crist Instrument). The recording chamber was placed overlying the cortical and striatal regions and was centrally positioned along the medial lateral axis. The calculated placement sites from magnetic resonance (MR) images are shown in Figure 2.

Activity of single units was recorded extracellularly with acutely placed bundles of Teflon insulated tungsten microwires (50 $\mu \mathrm{m}$ diameter; California Fine Wire) from OFC, ACC and dorsal striatum while monkeys were performing the task. Bundles consisting of four wires, were lowered through a $23 \mathrm{G}$ sharpened guide tube using either a manual (Crist Instrument) or electronically controlled microdrive (Plexon) to the target regions according to the MR images as previously described for microdialysis probe placement (Bradberry et al., 2000). Up to six independently placed bundles were used in a session. There was no prescreening for any selectivity of neurons. The standard procedure was to take $30 \mathrm{~min}$ to isolate units, then allow $30 \mathrm{~min}$ for stabilization before beginning the task. Neural activity was recorded using a Plexon Multichannel Acquisition Processor systems and sorted using Offline Sorter (Plexon). Further processing was conducted using Neuroexplorer (Nex Technologies) and Matlab (MathWorks).

Data analyses. To ensure that behavioral responding was consistent, we did not use six sessions during which the time to complete the contingency was 3 SDs greater than the mean (i.e., $>150 \mathrm{~s}$, leaving a total of 74 self-administration sessions, and 71 extinction sessions). We focused our analysis on neurons with a basal firing rate between 0.5 and $20 \mathrm{~Hz}$ as determined over a $12 \mathrm{~s}$ period before the onset of the discriminative cue. 

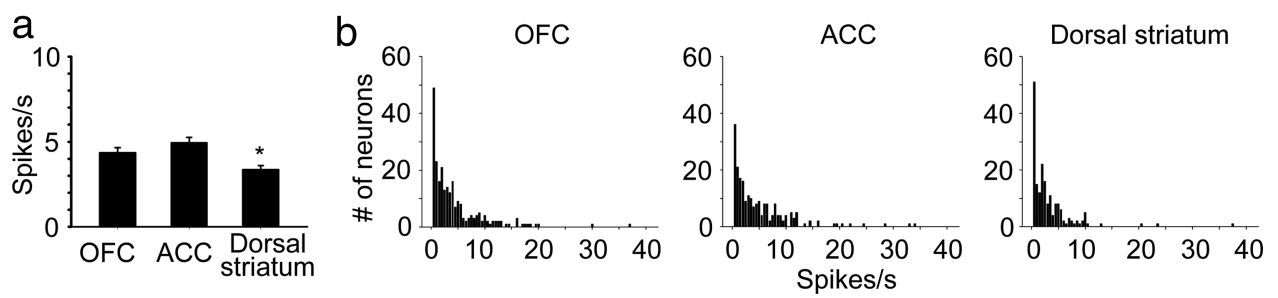

Figure 3. Regional comparison of basal firing rates. $\boldsymbol{a}$, Mean basal firing rate of analyzed neurons during the baseline period in each region \pm SEM. Dorsal striatum firing rate was significantly lower than both OFC and ACC. $\boldsymbol{b}$, Firing rate distribution for all neurons recorded in each region. Step size for histogram bars is $0.5 \mathrm{spikes} / \mathrm{s}$.
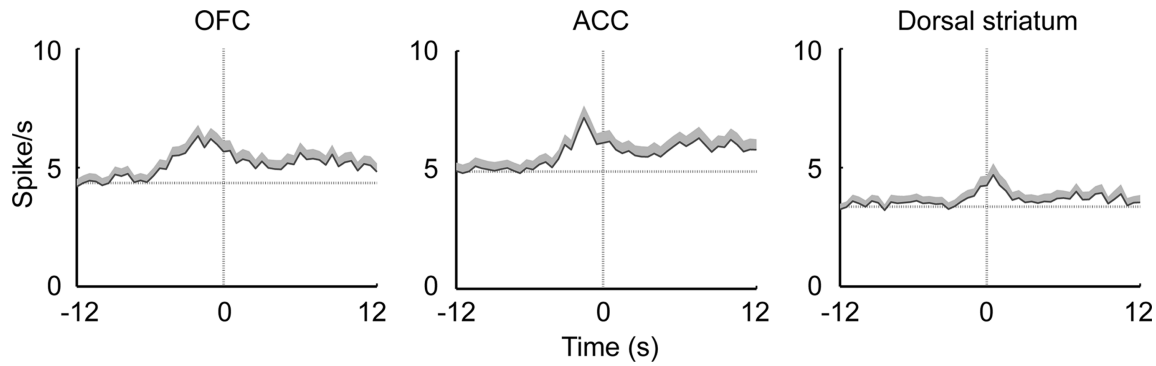

Figure 4. Firing was not tightly linked to lever pressing behavior. Each panel shows the mean firing rate plots by region, time locked to the time of the first lever press on each trial. Shaded area above each line is + SEM.

The lower cutoff ensured an adequate number of spikes for statistical comparison, while the upper cutoff avoided the excessive influence of a very few neurons on statistical comparisons because of their very high firing rates. The complete distribution of basal firing rates for each region is shown in Figure 3.

Mean firing rates for the population responses were calculated using a bin size of $0.5 \mathrm{~s}$. To subdivide the population response (see Fig. 5) (which represents the mean firing rate across all analyzed neurons) into population responses for neurons that increased or decreased their firing (see Fig. 8), the baseline firing rate ( $12 \mathrm{~s}$ before discriminative cue onset) was compared with the mean firing rate during the entire cue on period.

To determine whether neurons showed a significant response to a cue, the number of spikes during baseline ( $2 \mathrm{~s}$ before the onset of the discriminative cue) and $2 \mathrm{~s}$ after the onset of the discriminative cue or the discrete cue were compared (Mann-Whitney $U$ test, $p<0.05$ ). We chose the $2 \mathrm{~s}$ window to obtain a sufficient number of spikes for analysis before lever pressing behavior, which did not occur within this window on $>85 \%$ of trials (Fig. $1 c$ shows complete statistics for time to first lever press). We note also, as shown in Figure 4 that lever pressing behavior was not associated with sharp changes in firing in any region. A $\chi^{2}$ test was used for comparing regional differences in the proportion of responsive neurons. To compare changes in firing rate between regions during the last $2 \mathrm{~s}$ of the discrete cue, an absolute $Z$-score was used because of the large proportion of both increases and decreases in firing rate relative to baseline. $Z$-score was defined as the change from baseline as a multiple of the SD for that neuron.

To examine the time course of the neuronal differentiation between the discriminative cue and discrete cue, a sliding receiver operating characteristic (ROC) analysis was used. This measures the degree of overlap of the neuronal response distributions between two conditions. (For a brief description, see Wallis and Miller, 2003; Johnston et al., 2007.) We used area under the ROC curve as an index of differential response of a neuron between the two conditions (discriminative cue and discrete cue). In this analysis, a value of 0.5 indicates there is no differentiation between the two conditions, whereas a value of 1.0 indicates no overlap in the response distributions. We restricted our comparison to a $2 \mathrm{~s}$ period following onset of the discriminative cue and discrete cue. Neuronal responses in a $0.5 \mathrm{~s}$ window sliding in $0.01 \mathrm{~s}$ steps were compared between the two cues. To evaluate differences in the mean ROC value, a Bonferroni-corrected one-way ANOVA was used to determine regional differences in $0.5 \mathrm{~s}$ windows incremented at $0.01 \mathrm{~s}$ steps.

The time course of response to the discrete cue relative to precue baseline was also calculated using sliding ROC analysis. To calculate the
ROC values, a $0.5 \mathrm{~s}$ window was compared with each of the five $0.5 \mathrm{~s}$ windows comprising a $2.5 \mathrm{~s}$ baseline period before the discriminative cue. The ROC was the mean of those five comparisons. This was repeated for each $0.5 \mathrm{~s}$ window created by $0.01 \mathrm{~s}$ steps.

Data analysis for extinction. To compensate for the different number of trials per session, five trials (to match the number of cue presentations during self-administration sessions) were randomly selected from the total of 14-17 cue presentations in each extinction session. Whether the neuron had a significant response was then evaluated in the same way as described above, i.e., by Mann-Whitney comparison of the number of spikes in the $2 \mathrm{~s}$ after cue onset to the $2 \mathrm{~s}$ precue baseline. If that comparison indicated a significant response, then we counted the neuron as responsive to the discriminative cue. This process was repeated 100 times, and the median value for the proportion of responsive neurons is what was used for the extinction condition for comparison to the self-administration condition.

\section{Results}

A discriminative cue and discrete cue which had been associated with cocaine self-administration over a period of 4.5 years were used. A red lamp on an operant panel served as the discriminative cue indicating that an infusion of cocaine was available following a fixed ratio of 10 lever presses (FR10 contingency), after which, an $18 \mathrm{~s}$ infusion commenced to flush a bolus of cocaine $(0.1$ $\mathrm{mg} / \mathrm{ml}$ ) through the intravenous line. During the infusion, a green lamp on the operant panel was lit which served as the discrete cue (Fig. 1a). Thus, the discriminative cue was always present just before onset of the discrete cue, necessitating the use of a prediscriminative cue baseline. In each session, 5 cocaine infusions were available, separated by a $10 \mathrm{~min}$ imposed time out. The first four infusions were $0.1 \mathrm{mg} / \mathrm{kg}$ each, while the fifth was $0.5 \mathrm{mg} / \mathrm{kg}$. Given only one trial per session, it was not possible to evaluate whether cue responses differed for the $0.5 \mathrm{mg} / \mathrm{kg}$ infusion. We note that our measurements were not made at a time postinfusion when direct pharmacological effects of the higher dose would be apparent.

Consistent with well learned operant behavior, the response rate during the discriminative cue was significantly higher than during the baseline period (the $12 \mathrm{~s}$ before the onset of the discriminative cue) or during the discrete cue (Fig. $1 b$, one-way ANOVA, $\left.F_{(2,219)}=408, p=1.2 \times 10^{-74}\right)$. This demonstrates the salience of the discriminative cue, and the understanding by the monkeys that responding during the discrete cue had no consequence. The mean time to reach contingency (duration of discriminative cue) was $12.9 \pm 0.6 \mathrm{~s}$. Figure $1 c$ indicates complete statistics for the time to initiate the first lever press.

Neurons from OFC, ACC, and dorsal striatum were simultaneously recorded using up to six acutely placed, independently 
driven, four electrode arrays in two female rhesus monkeys during cocaine selfadministration. We recorded a total of 242, 218, and 185 well isolated neurons from OFC, ACC, and dorsal striatum, respectively (70, 26, and 39 from OFC, ACC, and dorsal striatum in animal A, and 172, 192, and 146 in animal B). A comparison of the mean basal firing rate of all neurons from each region used for the analyses is shown in Figure $3 a$. Values were significantly different between regions (one-way ANOVA, $F_{(2,476)}=6.65, p=$ $0.0014)$. Least significant difference (LSD) post hoc showed no difference between OFC and ACC, whereas basal firing rate in dorsal striatum was significantly lower than both OFC $(p=0.017)$ and ACC $(p=0.00032)$. We focused our analysis on neurons with a basal firing rate between 0.5 and $20 \mathrm{~Hz}$, yielding 188, 157, and 134 for the three regions, respectively, with the vast majority of exclusions due to neurons with firing rates below the minimum (Fig. $3 b$ ).

\section{Differential response of OFC to the two cues}

The discriminative cue signaled the immediate availability of cocaine upon completion of the lever press response requirement. As shown in Figure $1 b$, the discriminative cue clearly controlled operant responding. The discrete cue, in contrast, did not increase lever pressing above baseline. Thus, the two cues are associated with cocaine reward, but under quite distinct behavioral contingencies. Given the function of OFC in encoding the salience of a reward-associated stimulus into a goal that requires an instrumental action (Rolls and Grabenhorst, 2008), it could be predicted that the OFC would have a greater response to the discriminative cue than to the discrete cue. This was found to be the case. Figure 5 depicts the mean firing rate across all neurons analyzed in each region, referenced to cue onset for both the discriminative cue and the discrete cue. Comparing the mean firing rate during the beginning (first $2 \mathrm{~s}$ ) of the discriminative cue and discrete cue showed that OFC neuronal responses were significantly greater following the discriminative cue (paired $t$ test, $t_{(187)}=4.53, p=1.0 \times 10^{-5}$; Fig. 5 insets). In contrast, neither ACC nor dorsal striatum had differentially altered firing during the beginning of the cue on periods.

The greater mean firing rate in OFC during the discriminative cue could indicate a proportionately larger fraction of all neurons responding during the discriminative cue compared with the discrete cue. In support of this prediction, the proportion of neurons in OFC with a significant response to the discriminative cue was larger than to the discrete cue ( $\chi^{2}$ test, $p=0.0068$ for OFC), as shown in Figure 6, whereas the ACC and dorsal striatum showed no difference in proportion of neurons responding to the discriminative cue compared with the discrete cue.

As can be seen in the mean firing rate curves in Figure 5, the time at which the firing rate of OFC neurons was most different between the two cue types was just after onset. The time course of differentiation between the two cues was evaluated using ROC analysis as shown in Figure 7. ROC analysis indicates the proba-
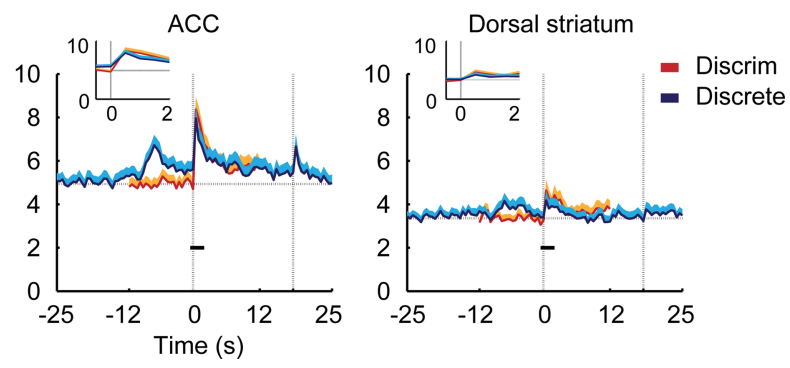

Figure 5. Mean firing rate plots for all analyzed neurons across regions. Onset of the discriminative cue and discrete cue was at $=0$. (Increases before time $=0$ for the discrete cue are due to the preceding discriminative cue.) The small black bars

Figure 6. Proportional responding of neurons in each region. The bars represent the proportion of neurons that showed a (he fomparison of the number of spikes in the baseline period ( 2 s before discriminative cue onset) with the first

bility of separating a neuronal response between two conditions, with a value of 0.5 indicating no differentiation, and 1.0 indicating complete differentiation (Wallis and Miller, 2003). The analysis was restricted to the neurons that had a statistically significant response to at least one of the two cues. The ranked ROC curves of individual neurons (Fig. 7a) demonstrate the time course of the probability of differentiation between the two cues. Comparison of the mean ROC values across all neurons in each region (Fig. $7 b$ ) confirms that it is very early after cue onset that the mean ROC value is greater in OFC than the other two regions (black dots mark bins with a significant difference between regions by one-way ANOVA with Bonferroni correction, $p<$ 0.00033; gray dots mark bins that differ between OFC and ACC, LSD post hoc, $p<0.05)$. The activity of a representative OFC neuron during presentation of the two cues is shown in Figure $7 c$.

To summarize the OFC pattern of response to the two cue types: by both mean firing rate across all neurons analyzed, and proportion of neurons responding, the OFC responds more strongly to the discriminative cue than to the discrete cue. Comparison of mean ROC values indicates that the distinction by OFC occurs very early after cue onset.

\section{Sustained response of the ACC to the discrete cue}

The ACC showed a very different pattern of response to the cues compared with the OFC. The neuronal response to the discriminative cue and the discrete cue did not differ either in mean firing rate (Fig. 5) or proportion of neurons that were initially responsive to the discriminative cue and discrete cue (Fig. 6). What most distinguished the ACC was a pattern of sustained neuronal response throughout the discrete cue. This can be seen in the mean firing rate plot in Figure 5 in that the mean firing rate remains further above baseline throughout the entire cue on period, com- 

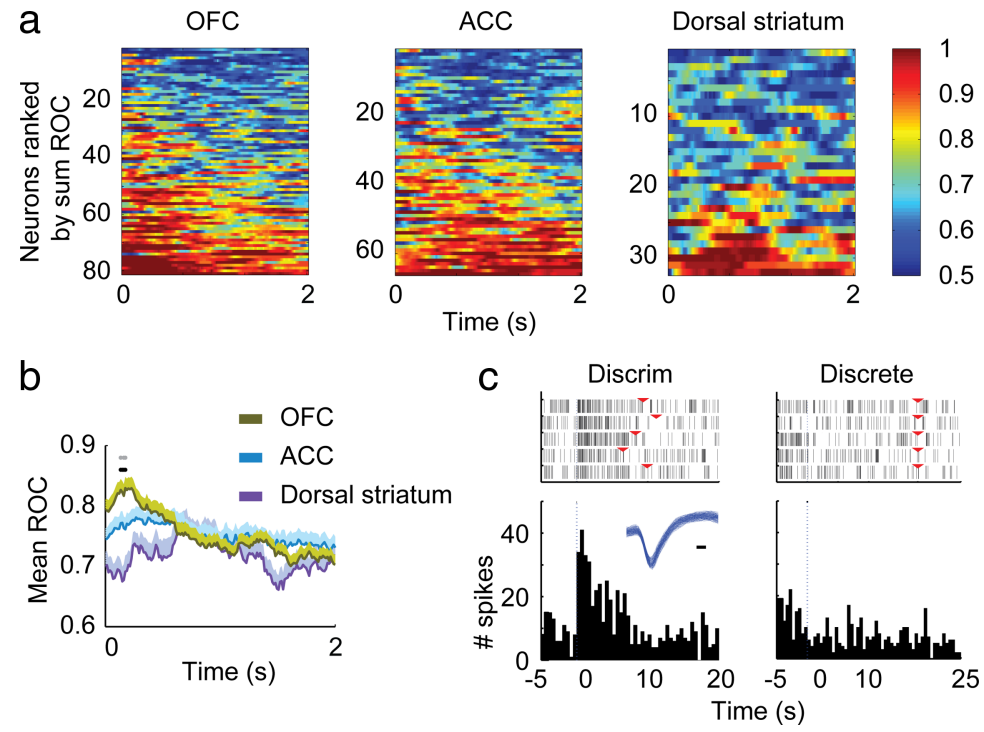

Figure 7. Selectivity between the discriminative cue and discrete cue using ROC analysis. Only responsive neurons (determined as in Fig. 6) to either one or both cue types were used for this analysis. $\boldsymbol{a}$, A sliding window ( $0.5 \mathrm{~s}$ wide, $0.01 \mathrm{~s}$ steps) was used to compare the neuronal responses during the first $2 \mathrm{~s}$ of the two cues. Figures were plotted by ordering neurons from top to bottom by increasing sum of the ROC value. $\boldsymbol{b}$, Mean ROC values for all neurons from $\boldsymbol{a}$. Black dots indicate the time periods of differences among regions, the gray dots are the points that showed significant difference between $\mathrm{OFC}$ and $\mathrm{ACC}$. c, Example of an OFC neuron which showed more responses to the discriminative cue (left) compared with responses to discrete cue (right). Inset shows the waveform of the neuron, black bar $=0.1 \mathrm{~ms}$. The red triangles on the raster plots indicate the onset of the discrete cue on the left panel, and offset of the discrete cue on the right panel.
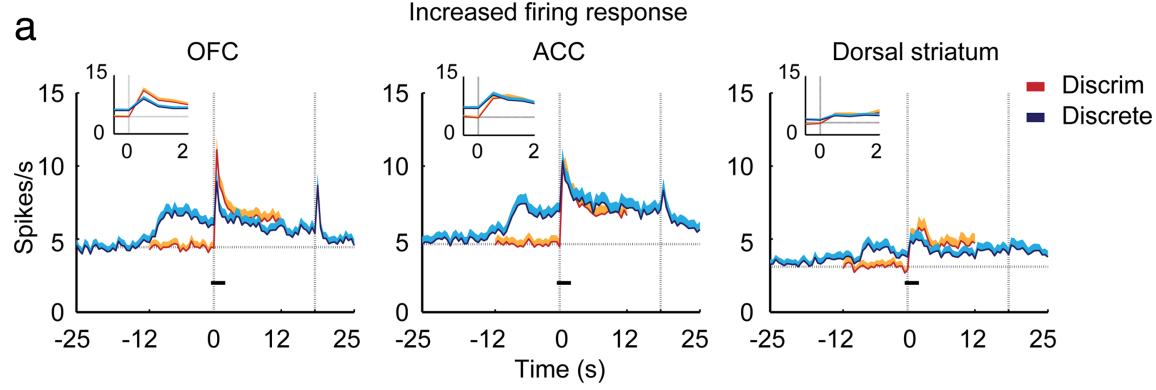

b

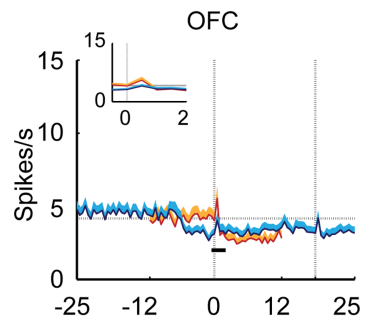

Decreased firing response
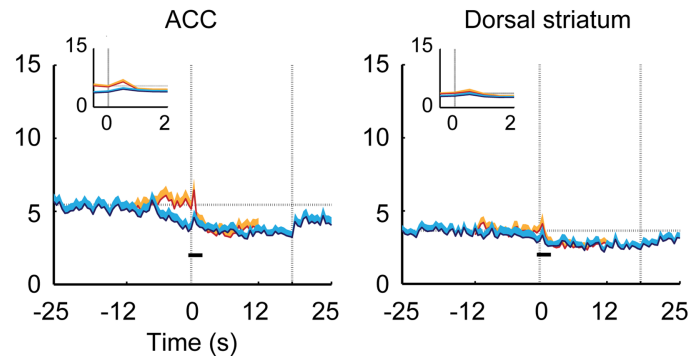

Figure 8. Population responses of all neurons from Figure 5 , subdivided according to whether their firing rate increased $(\boldsymbol{a})$ or decreased $(\boldsymbol{b})$ relative to baseline following cue onset. Note the more sustained response of ACC neurons that either increased or decreased firing following onset of the discrete cue. For the discriminative cue, the breakdown by region was: OFC, 127 increased, 61 decreased; ACC, 102 increased, 55 decreased; STR, 72 increased, 62 decreased. For the discrete cue, the breakdown by region was: $0 F(116$ increased, 72 decreased; ACC, 92 increased, 65 decreased; dorsal striatum, 74 increased, 60 decreased.

pared with either OFC or dorsal striatum. However, the mean firing rate plot somewhat obscures this effect because neurons showed both increases and decreases in firing rate in response to the cue. Long-lasting changes in ACC neuronal firing are much more apparent when broken down according to the direction of firing rate change (Fig. 8), in which all of the neurons analyzed to produce the population response shown in Figure 5, are broken down by direction of firing rate change. There, it can be seen that the responses of both excited and inhibited neurons to the discrete cue are clearly more extensive and prolonged than in the other regions.

The pattern of sustained response in ACC is also demonstrated by a comparison of the proportion of neurons that have a significantly different firing rate (from baseline) during the beginning (first $2 \mathrm{~s}$ ) and end (last $2 \mathrm{~s}$ ) of the discrete cue period. Figure 6 demonstrates that in the ACC, there was no difference in the proportion of neurons with a firing rate different from baseline between the beginning and end of the discrete cue $\left(\chi^{2}\right.$ test, $p=0.24)$. In contrast, a significant decrease in the proportion of responsive neurons between the beginning and end of the cue is seen for both the OFC and dorsal striatum (Fig. 6, $\chi^{2}$ test, $p=0.017$ for OFC, $p=0.035$ for dorsal striatum). In addition to the ACC not showing a change in proportion of responsive neurons between the beginning and end of the discrete cue, two additional comparisons indicate that the ACC remains more responsive at the end of the discrete cue than either the OFC or dorsal striatum. First, a $\chi^{2}$ test indicates that there is a regional difference in proportion of responsive neurons during the end of the discrete cue, relative to baseline (overall effect, $p=0.00096$; ACC vs OFC, $p=0.028$, ACC vs dorsal striatum, $p=0.00031$, OFC vs dorsal striatum, $p=0.074$ ). A second positive indicator of a regional difference is ANOVA of the absolute $Z$-scores across regions (overall comparison, $F_{(2,476)}=$ $4.5, p=0.012$; LSD post hoc comparison: ACC vs OFC, $p=0.026$; ACC vs dorsal striatum, $p=0.0045$; OFC vs dorsal striatum, $p=0.40)$.

ROC analysis (Fig. 9a,b) reveals not only the greater number of individual neurons in ACC that differentiate discrete cue from baseline, but also how clearly the neuronal response in ACC is linked to the actual light-on period. This is shown by the sharp break at the 17.5/18 s window (marked by the two vertical lines) and can be seen as well in the response of the representative neuron shown in Figure $9 c$. Comparison of the mean ROC values across all neurons in each region (Fig. $9 b$ ) confirms that the increased neuronal response extends throughout the light-on period (by one-way ANOVA with Bonferroni correction, $p<2.0 \times 10^{-5}$; gray dots mark bins that differ between OFC and ACC, LSD post hoc, $p<$ $0.05)$. The ROC analysis reveals the pattern of differentiation of each analyzed neuron, and thereby demonstrates that a separate population is not becoming active just before cue offset. This is important because it is likely that subjective effects from the 
cocaine are beginning to occur toward the end of the discrete cue. The entry of cocaine into the animal was somewhat delayed from cue onset, given that a bolus was placed in the intravenous line some distance from the animal. Visual inspection of a bolus of dye using an identical set of lines and vascular access port showed that the $0.1 \mathrm{ml}$ bolus placed in the line visibly exited the catheter tip across an interval from 7.5 to $12.5 \mathrm{~s}$ of the infusion. Based on human reports (Evans et al., 1996), cocaine subjective effects would have begun within a few seconds after exiting the catheter tip, and thus direct pharmacological effects should not have begun until the latter portion of the cue on period. Therefore, a developing subjective sensation of cocaine's effects could not be the cause of the sustained response in the ACC.

To summarize the ACC pattern of response to the two cue types, there is not a difference in initial response to the discriminative cue and discrete cue as shown by mean firing rate and proportion of neurons responding. The ACC neuronal response extended throughout the entire discrete cue period as shown by no change in the proportion of responsive neurons between the beginning and end of the cue period, a difference between regions in the proportion of responsive neurons at the end of the cue period, and a difference between regions in absolute $Z$-scores of the firing rate at the end of the cue period.

\section{Diminished dorsal striatum responses relative to OFC and ACC}

Compared with the two cortical regions examined, the dorsal striatum was less responsive to drug-associated cues. This can be seen in the mean firing rate graphs in Figure 5, and in the proportion of responsive neurons to each cue type in Figure 6. When we compared the proportion of responsive neurons across the three regions, there was a significant difference among regions for both the discriminative cue and discrete cue $(p=0.00021$, and $p=0.031$, respectively). More specifically, in response to the discriminative cue, dorsal striatum had a lower proportion of responsive neurons than both OFC $\left(p=5.0 \times 10^{-5}\right)$ and $\operatorname{ACC}(p=0.0014)$. In response to the first $2 \mathrm{~s}$ of the discrete cue, the dorsal striatum response was smaller than ACC ( $p=0.0083)$, and not different from OFC ( $p=0.088$, all comparisons by $\chi^{2}$ test $)$.

\section{Impact of extinction on neuronal responding}

To confirm that neuronal responding to the cues was related to their reward significance, an extinction procedure was used in animal B. Substitution of saline for cocaine gradually reduced lever pressing behavior in response to discriminative cue presentation, though it took 24 sessions for the rate of successful completion of the FR10 contingency to drop below $80 \%$ over two
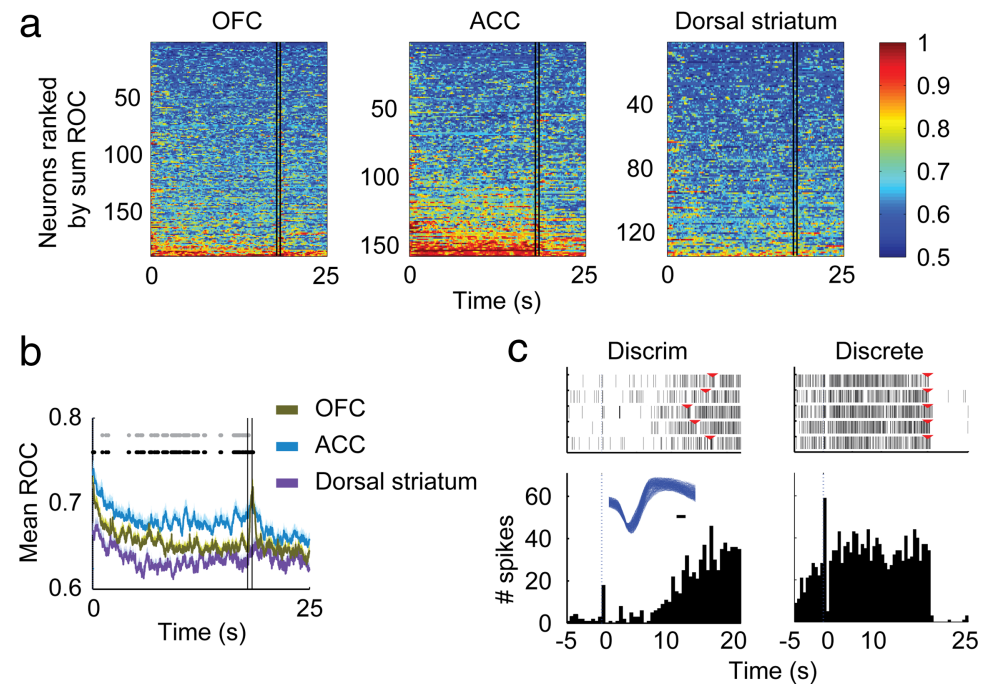

Figure 9. Selectivity between the discrete cue and baseline using ROC analysis demonstrating the sustained response of ACC neurons to the discrete cue. $\boldsymbol{a}$, A sliding window ( $0.5 \mathrm{~s}$ wide, $0.01 \mathrm{~s}$ steps) was used to compare the neuronal responses to baseline. top to bottom by increasing sum of the ROC value. The two vertical black lines (at $T=17.5 \mathrm{~s}$ and $\mathrm{T}=18 \mathrm{~s}$ ) represent the the discrete cue (right). Inset shows the waveform of the neuron, black bar $=0.1 \mathrm{~ms}$. The red triangles on the raster plots indicate a

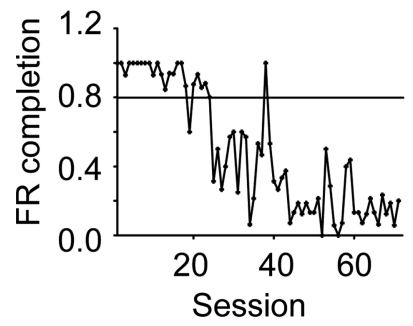

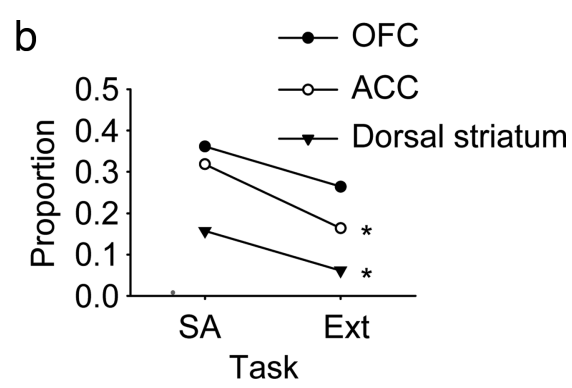

Figure 10. Behavior and unit firing during cocaine extinction. $\boldsymbol{a}$, FR completion represents the fraction of trials in which the FR10 lever press contingency was completed, resulting in saline infusion. A total of 71 extinction sessions were conducted. The horizontal line represents the cutoff of $<80 \%$ of FR contingencies met over 2 successive sessions used for $\boldsymbol{b}$. Although lever pressing behavior was not completely extinguished, it was reduced across extinction sessions (linear regression, $p=1.0 \times 10^{22}$ ). Comparison of proportion of responsive neurons between sessions during cocaine self-administration (SA) and during extinction (Ext) according to region. Sessions after the criterion indicated on $\boldsymbol{a}$ (session 25-71) were used to calculate the proportion of responsive neurons during extinction. A significant difference was observed in ACC and dorsal striatum by $\chi^{2}$ test comparing SA to Ext ( $p=0.0010$ for ACC, $p=0.0385$ for dorsal striatum). OFC was not significantly different ( $p=0.07$ ).

consecutive sessions (Fig. 10a). Using all sessions (25-71) past that criterion, the proportion of responsive neurons before and after partial extinction were compared (Fig. 10b). There was a significant decrease in ACC and dorsal striatum $\left(\chi^{2}\right.$ test, $p=$ 0.0010 for ACC, $p=0.0385$ for dorsal striatum), however, in the OFC, the proportion of responsive neurons was not significantly different $(p=0.07)$.

\section{Discussion}

We observed that in OFC and ACC, two cortical regions prominently implicated in addiction, there was a profound engagement of neuronal activity by drug-associated cues. Selective functionality of the two cortical regions was apparent in that the OFC was more engaged by the discriminative cue relative to the discrete cue. In contrast, while not differing in initial response to the two cue types, the ACC displayed a much more sustained response 
throughout the entire discrete cue associated with cocaine infusion. This study is the first to report cortical neuronal responses to drug-associated cues in nonhuman primates with a long history of conditioning that is typical of addiction in humans. These results define, at a neuronal activity level, the engagement of two key cortical regions by distinct types of cues known to differentially control drug reinforced behavior in animals.

The substantial primate electrophysiology literature on neuronal activity in cortical regions such as OFC and ACC during non-drug reward tasks has significantly shaped the interpretation of human imaging findings related to cue reactivity and cognitive dysfunction associated with addiction. The electrophysiological studies of dopamine neuronal responses in primates (Mirenowicz and Schultz, 1996) to rewards and reward cues have also been highly influential in guiding an understanding of how the dopaminergic properties of drugs of abuse contribute to their reinforcing effects. However, despite the contributions of primate electrophysiolgical studies to basic reward mechanisms, preclinical electrophysiological studies of neuronal activity responses to drugs of abuse and associated cues have, with one exception (Bowman et al., 1996), been conducted in the rodent. Within the rodent literature, there is relatively little work on electrophysiological measures of cortical neuronal activity responses to drugassociated cues (Chang et al., 1997, 1998, 2000; Sun and Rebec, 2006). Given that clinical imaging methods assess measures dependent on blood flow and metabolism which are influenced more by local synaptic processing than action potential generation (Goense and Logothetis, 2008), the current results complement imaging-based measures by assessing the actual neuronal firing that results from that processing. Thus, studying cortical single-unit activity in primates wherein the long term nature of human addiction can be modeled fills a significant gap in addiction research.

We used an unbiased sampling of neurons within each region, as there was no attempt to assess any task related responding before cue exposure. Thus, we are reporting comparisons of integrated responses between regions. We chose to use highly reinforcing doses of cocaine for these studies to more closely mimic actual patterns of consumption. Each infusion has an extended duration of action determined by the rate of metabolism $\left(t_{1 / 2}\right.$ for elimination in rhesus monkeys is $30-45 \mathrm{~min}$ ) and acute tolerance to the pharmacological effects (Bradberry, 2000). Therefore, the number of trials that could be conducted within a session was limited. Consequently, we did not attempt to explicitly control for neural responses that could be selective for visual characteristics of stimuli such as color, size, etc. within session as done in the elegant studies from the many primate electrophysiological labs studying reward related function in these regions (Apicella et al., 1992; Schultz et al., 1992; Bowman et al., 1996; Roesch and Olson, 2004; Padoa-Schioppa and Assad, 2006; Rolls and Grabenhorst, 2008). Though previous work has shown that some unit activity in striatum is linked to movement execution such as in the case of visual saccades (Hikosaka et al., 1989), a general conclusion from the body of work on both striatal and cortical responses to instructional and reward-associated cues is that responding is primarily associated with their reward relevance rather than sensory processing or motor response preparation. Given that we used only five trials per session, the high proportion of statistically significantly responsive cortical neurons we observed is an indicator of how strongly these cues engaged the OFC and ACC.

OFC had a significantly greater response to the discriminative cue than the discrete cue, unlike the other regions examined.
Previous work has shown that OFC is involved in reward evaluation and initiation of reward guided responses (Roesch and Olson, 2004; Padoa-Schioppa and Assad, 2006; Rolls and Grabenhorst, 2008). Our observation of preferential engagement of OFC by a discriminative cue suggests a role for OFC in relapse triggered by environmental cues indicating drug availability, given that discriminative cues support more drug seeking than discrete cues when presented noncontingently in animal models (Di Ciano and Everitt, 2003; Yun and Fields, 2003). During the extinction process which one animal underwent, the OFC response was the most resistant to extinction. This was surprising, because normally, one of the key functions of the OFC is an ability to rapidly adapt to changing reward contingencies and preferences following satiety (Rolls and Grabenhorst, 2008). However, chronic exposure to cocaine appears to seriously diminish this adaptive flexibility of stimulus reward associations (Schoenbaum and Shaham, 2008) in OFC. Our results are consistent with these observations. It appears plausible that a chronically enduring responsiveness to a discriminative cue by the OFC could represent a resilient entryway for discriminative cues to trigger relapse long after establishment of abstinence.

The other striking regionally selective cortical response was the prolonged engagement of neurons in ACC during the discrete cue which ceased when it was turned off. A prolonged response is consistent with the established role of the ACC in reward expectation and in guiding behavior for obtaining rewards. However, this involvement of the ACC in response selection and evaluation of outcome has generally been established over short term action outcome contingencies (Shidara and Richmond, 2002). In the present case, the ACC engagement was maintained even when responses were no longer necessary. ACC receives less sensory input than OFC, and is strongly connected with higher level motor processing centers (Carmichael and Price, 1995), consistent with the ACC being a key node in a cognitive control network devoted to response preparation (Cole and Schneider, 2007). What we observed was a high degree of activation throughout the discrete cue, despite the fact that it specifically signaled that no behavioral response was needed (and virtually none were made). This enduring engagement of ACC by discrete drug cues could be a mechanism through which those external cues maintain their prominence within motivational and motor planning networks.

The proportion of neurons engaged and the duration of the population responses to cues in the cortical regions far surpassed those in dorsal striatum. This is particularly significant in relation to what neural processes might be sustaining an enduring state of mind such as craving. Importantly, these are also cortical regions shown clinically to be highly responsive to drug-associated cues. In contrast to the cortex, striatal regions generally do not show activation with cue exposure (Grant et al., 1996; Maas et al., 1998; Wexler et al., 2001) (but see Garavan et al., 2000), unless the cues were based on internal imagery (Kilts et al., 2001). The dorsal striatum wherein we recorded neuronal responses is heavily innervated by dorsolateral prefrontal cortex and to a lesser extent by ACC and OFC (Haber et al., 2006). It is considered to be a likely site of convergence of a range of cortical inputs placing it in an excellent position to influence reward-based learning (Haber et al., 2006). A shift of mediation of drug reinforcement from ventral to more dorsal striatum has been proposed to accompany the transition from voluntary to compulsive drug consumption (Everitt et al., 2008). It is difficult to assess the functional homology of the dorsal striatal region we sampled with that from rodent-based preclinical studies given substantial differences in cortical inputs. However, if dorsal striatal neuronal responses are 
mediating behavioral responding to drug cues in these monkeys with an extensive history of self-administration, they are doing so with relatively less engagement than the cortical areas sampled.

We have examined neuronal activity responses to drug cues in animals with a long history of exposure to those cues. However, comparisons with non-drug reward-associated cues were not made. Consequently, we do not infer that the selective regional patterns of responding we observed only apply to drug rewards, nor that they necessarily are the key mechanisms underlying addiction or relapse. However, the present report does complement the substantial clinical literature indicating greater cingulate and orbitofrontal cortical reactivity to drug cues relative to striatum (Grant et al., 1996; Maas et al., 1998; Garavan et al., 2000; Wexler et al., 2001; Kosten et al., 2006; Goldstein et al., 2007, 2009). It also establishes unique functionality associated with the different prefrontal cortical regions in response to environmental cues that drive distinct aspects of drug-reinforced behavior in animals. In so doing, it will help to lay the foundation for future research to identify cognitive control mechanisms whereby drug cues exert such a profound influence over decisions and behavior, and potential neuronal activity metrics for pharmacotherapy development.

\section{References}

Apicella P, Scarnati E, Ljungberg T, Schultz W (1992) Neuronal activity in monkey striatum related to the expectation of predictable environmental events. J Neurophysiol 68:945-960.

Aron JL, Paulus MP (2007) Location, location: using functional magnetic resonance imaging to pinpoint brain differences relevant to stimulant use. Addiction 102 [Suppl 1]:33-43.

Bowman EM, Aigner TG, Richmond BJ (1996) Neural signals in the monkey ventral striatum related to motivation for juice and cocaine rewards. J Neurophysiol 75:1061-1073.

Bradberry CW (2000) Acute and chronic dopamine dynamics in a nonhuman primate model of recreational cocaine use. J Neurosci 20:7109-7115.

Bradberry CW, Barrett-Larimore RL, Jatlow P, Rubino SR (2000) Impact of self-administered cocaine and cocaine cues on extracellular dopamine in mesolimbic and sensorimotor striatum in rhesus monkeys. J Neurosci 20:3874-3883.

Carmichael ST, Price JL (1995) Sensory and premotor connections of the orbital and medial prefrontal cortex of macaque monkeys. J Comp Neurol 363:642-664.

Chang JY, Sawyer SF, Paris JM, Kirillov A, Woodward DJ (1997) Single neuronal responses in medial prefrontal cortex during cocaine selfadministration in freely moving rats. Synapse 26:22-35.

Chang JY, Janak PH, Woodward DJ (1998) Comparison of mesocorticolimbic neuronal responses during cocaine and heroin self-administration in freely moving rats. J Neurosci 18:3098-3115.

Chang JY, Janak PH, Woodward DJ (2000) Neuronal and behavioral correlations in the medial prefrontal cortex and nucleus accumbens during cocaine self-administration by rats. Neuroscience 99:433-443.

Cole MW, Schneider W (2007) The cognitive control network: Integrated cortical regions with dissociable functions. Neuroimage 37:343-360.

Di Ciano P, Everitt BJ (2003) Differential control over drug-seeking behavior by drug-associated conditioned reinforcers and discriminative stimuli predictive of drug availability. Behav Neurosci 117:952-960.

Evans SM, Cone EJ, Henningfield JE (1996) Arterial and venous cocaine plasma concentrations in humans: relationship to route of administration, cardiovascular effects and subjective effects. J Pharmacol Exp Ther 279:1345-1356

Everitt BJ, Robbins TW (2000) Second-order schedules of drug reinforcement in rats and monkeys: measurement of reinforcing efficacy and drugseeking behaviour. Psychopharmacologia 153:17-30.

Everitt BJ, Belin D, Economidou D, Pelloux Y, Dalley JW, Robbins TW (2008) Review. Neural mechanisms underlying the vulnerability to develop compulsive drug-seeking habits and addiction. Philos Trans R Soc Lond B Biol Sci 363:3125-3135.

Garavan H, Hester R (2007) The role of cognitive control in cocaine dependence. Neuropsychol Rev 17:337-345.
Garavan H, Pankiewicz J, Bloom A, Cho JK, Sperry L, Ross TJ, Salmeron BJ, Risinger R, Kelley D, Stein EA (2000) Cue-induced cocaine craving: neuroanatomical specificity for drug users and drug stimuli. Am J Psychiatry 157:1789-1798.

Goense JB, Logothetis NK (2008) Neurophysiology of the BOLD fMRI signal in awake monkeys. Curr Biol 18:631-640.

Goldberg SR, Spealman RD, Goldberg DM (1981) Persistent behavior at high rates maintained by intravenous self-administration of nicotine. Science 214:573-575.

Goldstein RZ, Tomasi D, Rajaram S, Cottone LA, Zhang L, Maloney T, Telang F, Alia-Klein N, Volkow ND (2007) Role of the anterior cingulate and medial orbitofrontal cortex in processing drug cues in cocaine addiction. Neuroscience 144:1153-1159.

Goldstein RZ, Alia-Klein N, Tomasi D, Carrillo JH, Maloney T, Woicik PA, Wang R, Telang F, Volkow ND (2009) Anterior cingulate cortex hypoactivations to an emotionally salient task in cocaine addiction. Proc Natl Acad Sci U S A 106:9453-9458.

Grant S, London ED, Newlin DB, Villemagne VL, Liu X, Contoreggi C, Phillips RL, Kimes AS, Margolin A (1996) Activation of memory circuits during cue-elicited cocaine craving. Proc Natl Acad Sci U S A 93: $12040-12045$.

Haber SN, Kim KS, Mailly P, Calzavara R (2006) Reward-related cortical inputs define a large striatal region in primates that interface with associative cortical connections, providing a substrate for incentive-based learning. J Neurosci 26:8368-8376.

Hikosaka O, Sakamoto M, Usui S (1989) Functional properties of monkey caudate neurons. I. Activities related to saccadic eye movements. J Neurophysiol 61:780-798.

Iyer RN, Nobiletti JB, Jatlow PI, Bradberry CW (1995) Cocaine and cocaethylene: effects on extracellular dopamine in the primate. Psychopharmacology (Berl) 120:150-155.

Johnston K, Levin HM, Koval MJ, Everling S (2007) Top-down controlsignal dynamics in anterior cingulate and prefrontal cortex neurons following task switching. Neuron 53:453-462.

Kalivas PW, Volkow ND (2005) The neural basis of addiction: a pathology of motivation and choice. Am J Psychiatry 162:1403-1413.

Kilts CD, Schweitzer JB, Quinn CK, Gross RE, Faber TL, Muhammad F, Ely TD, Hoffman JM, Drexler KP (2001) Neural activity related to drug craving in cocaine addiction. Arch Gen Psychiatry 58:334-341.

Kosten TR, Scanley BE, Tucker KA, Oliveto A, Prince C, Sinha R, Potenza MN, Skudlarski P, Wexler BE (2006) Cue-induced brain activity changes and relapse in cocaine-dependent patients. Neuropsychopharmacology 31:644-650.

Maas LC, Lukas SE, Kaufman MJ, Weiss RD, Daniels SL, Rogers VW, Kukes TJ, Renshaw PF (1998) Functional magnetic resonance imaging of human brain activation during cue-induced cocaine craving. Am J Psychiatry 155:124-126.

McLaughlin J, See RE (2003) Selective inactivation of the dorsomedial prefrontal cortex and the basolateral amygdala attenuates conditioned-cued reinstatement of extinguished cocaine-seeking behavior in rats. Psychopharmacology (Berl) 168:57-65.

Mirenowicz J, Schultz W (1996) Preferential activation of midbrain dopamine neurons by appetitive rather than aversive stimuli. Nature 379:449-451.

Neisewander JL, Baker DA, Fuchs RA, Tran-Nguyen LT, Palmer A, Marshall JF (2000) Fos protein expression and cocaine-seeking behavior in rats after exposure to a cocaine self-administration environment. J Neurosci 20:798-805.

Padoa-Schioppa C, Assad JA (2006) Neurons in the orbitofrontal cortex encode economic value. Nature 441:223-226.

Passingham R (2009) How good is the macaque monkey model of the human brain? Curr Opin Neurobiol 19:6-11.

Robbins TW, Ersche KD, Everitt BJ (2008) Drug addiction and the memory systems of the brain. Ann N Y Acad Sci 1141:1-21.

Roesch MR, Olson CR (2004) Neuronal activity related to reward value and motivation in primate frontal cortex. Science 304:307-310.

Rolls ET, Grabenhorst F (2008) The orbitofrontal cortex and beyond: from affect to decision-making. Prog Neurobiol 86:216-244.

Rushworth MF, Behrens TE, Rudebeck PH, Walton ME (2007) Contrasting roles for cingulate and orbitofrontal cortex in decisions and social behaviour. Trends Cogn Sci 11:168-176. 
Schoenbaum G, Shaham Y (2008) The role of orbitofrontal cortex in drug addiction: a review of preclinical studies. Biol Psychiatry 63:256-262.

Schultz W, Apicella P, Scarnati E, Ljungberg T (1992) Neuronal activity in monkey ventral striatum related to the expectation of reward. J Neurosci 12:4595-4610.

Shidara M, Richmond BJ (2002) Anterior cingulate: single neuronal signals related to degree of reward expectancy. Science 296:1709-1711.

Sun W, Rebec GV (2006) Repeated cocaine self-administration alters processing of cocaine-related information in rat prefrontal cortex. J Neurosci 26:8004-8008.

Tokuno HA, Bradberry CW, Everill B, Agulian SK, Wilkes S, Baldwin RM, Tamagnan GD, Kocsis JD (2004) Local anesthetic effects of cocaethylene and isopropylcocaine on rat peripheral nerves. Brain Res 996: $159-167$.
Wallis JD, Miller EK (2003) Neuronal activity in primate dorsolateral and orbital prefrontal cortex during performance of a reward preference task. Eur J Neurosci 18:2069-2081.

Weiss F, Maldonado-Vlaar CS, Parsons LH, Kerr TM, Smith DL, Ben-Shahar O (2000) Control of cocaine-seeking behavior by drug-associated stimuli in rats: effects on recovery of extinguished operant-responding and extracellular dopamine levels in amygdala and nucleus accumbens. Proc Natl Acad Sci U S A 97:4321-4326.

Wexler BE, Gottschalk CH, Fulbright RK, Prohovnik I, Lacadie CM, Rounsaville BJ, Gore JC (2001) Functional magnetic resonance imaging of cocaine craving. Am J Psychiatry 158:86-95.

Yun IA, Fields HL (2003) Basolateral amygdala lesions impair both cue- and cocaine-induced reinstatement in animals trained on a discriminative stimulus task. Neuroscience 121:747-757. 